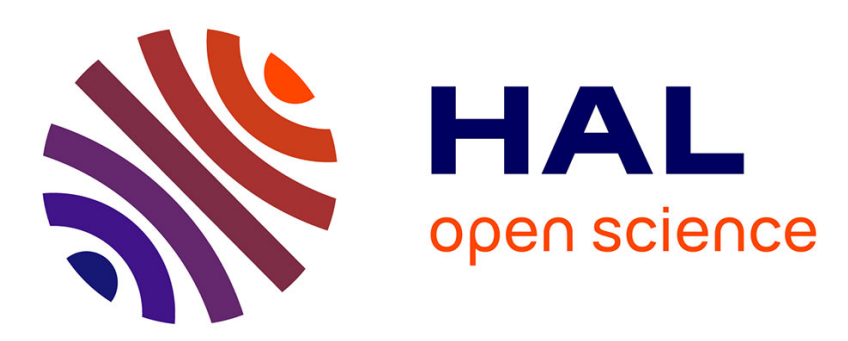

\title{
Heliolatitudinal and time variations of the solar wind mass flux: inferences from the backscattered solar Lyman-alpha intensity maps
}

Olga Katushkina, V. V. Izmodenov, Eric Quémerais, Justyna M. Sokól

\section{- To cite this version:}

Olga Katushkina, V. V. Izmodenov, Eric Quémerais, Justyna M. Sokól. Heliolatitudinal and time variations of the solar wind mass flux: inferences from the backscattered solar Lyman-alpha intensity maps. Journal of Geophysical Research Space Physics, 2013, 118 (6), pp.2800-2808. 10.1002/jgra.50303 . hal-00819670

\section{HAL Id: hal-00819670 \\ https://hal.science/hal-00819670}

Submitted on 27 Aug 2020

HAL is a multi-disciplinary open access archive for the deposit and dissemination of scientific research documents, whether they are published or not. The documents may come from teaching and research institutions in France or abroad, or from public or private research centers.
L'archive ouverte pluridisciplinaire HAL, est destinée au dépôt et à la diffusion de documents scientifiques de niveau recherche, publiés ou non, émanant des établissements d'enseignement et de recherche français ou étrangers, des laboratoires publics ou privés. 


\title{
Heliolatitudinal and time variations of the solar wind mass flux: Inferences from the backscattered solar Lyman-alpha intensity maps
}

\author{
O. A. Katushkina, ${ }^{1,2}$ V. V. Izmodenov, ${ }^{1,2,3}$ E. Quemerais, ${ }^{4}$ and J. M. Sokół ${ }^{5}$ \\ Received 27 December 2012; revised 17 April 2013; accepted 28 April 2013; published 7 June 2013.
}

[1] Recently Sokół et al. (2012) have presented a reconstruction of heliolatitudinal and time variations of the solar wind speed and density. Method of the reconstruction was based on the following: (i) measurements of the interplanetary scintillations, (ii) OMNI-2 solar wind data in the ecliptic plane, and (iii) Ulysses solar wind data out of the ecliptic plane. In this paper we use hydrogen charge exchange rates derived from their results as input parameters to calculate the interstellar hydrogen distribution in the heliosphere in the frame of our 3-D time-dependent kinetic model. The hydrogen distribution is then used to calculate the backscattered solar Lyman-alpha intensity maps. The theoretical Lyman-alpha maps are compared with the SOHO/SWAN measurements during maximum and minimum of the solar cycle activity. We found that in the solar minimum there is a quite good agreement between the model results and the SWAN data, but in the solar maximum sky maps of the Lyman-alpha, intensities are qualitatively different for the model results and observations. Physical reasons of the differences are discussed.

Citation: Katushkina, O. A., V. V. Izmodenov, E. Quemerais, and J. M. Sokół (2013), Heliolatitudinal and time variations of the solar wind mass flux: Inferences from the backscattered solar Lyman-alpha intensity maps, J. Geophys. Res. Space Physics, 118, 2800-2808, doi:10.1002/jgra.50303.

\section{Introduction}

[2] The knowledge of the temporal and heliolatitudinal variations of the solar wind parameters (particularly density and speed) is essential for both global modeling of the 3-D heliosphere including its boundaries and analysis of related spacecraft data (e.g., from IBEX and Voyagers 1 and 2). Numerous measurements of the solar wind parameters were made in the ecliptic plane during several decades, but only Ulysses spacecraft observed the solar wind out of the ecliptic plane [McComas et al., 2000] and provided direct information on the heliolatitudinal dependence of the solar wind parameters.

[3] Simultaneous in situ observations of the solar wind at all heliolatitudes are not possible, and indirect methods should be applied in order to obtain the time evolution of the 3-D solar wind structure.

[4] Essential indirect information on spatial and temporal variations of the solar wind is based on remote-

\footnotetext{
${ }^{1}$ Space Research Institute, Russian Academy of Sciences, Moscow, Russia.

${ }^{2}$ Department of Mechanics and Mathematics, Lomonosov Moscow State University, Moscow, Russia.

${ }^{3}$ Institute for Problems in Mechanics, Russian Academy of Sciences, Moscow, Russia.

${ }^{4}$ LATMOS, Paris, France.

${ }^{5}$ Space Research Centre, Polish Academy of Sciences, Warsaw, Poland.

Corresponding author: O. A. Katushkina, Space Research Institute, Russian Academy of Sciences, 84/32 Profsoyuznaya Str., Moscow 117997, Russia. (okat@iki.rssi.ru)

(C)2013. American Geophysical Union. All Rights Reserved. 2169-9380/13/10.1002/jgra.50303
}

sensing radio-observations of interplanetary scintillations (IPS). Computer Assisted Tomography (CAT) technique allows to obtain the solar wind speed from the IPS data [e.g., Jackson et al., 1998; Tokumaru et al., 2012; Manoharan, 2012]. Based on IPS data from Solar-Terrestrial Environmental Laboratory (STEL) at Nagoya University in Japan, Sokót et al. [2012] presented one year averaged solar wind speed as a function of heliolatitude for two last solar cycles (from 1990 to 2011).

[5] In addition to the velocity, Sokót et al. [2012] reconstructed time and heliolatitudinal variations of the solar wind number density at 1 AU. This reconstruction was based on in situ data from OMNI-2 database (http://omniweb.gsfc.nasa.gov/) [King and Papitashvili, 2005] that provides the solar wind parameters in the ecliptic plane during several decades, and in situ Ulysses solar wind data out of the ecliptic plane [McComas et al., 2003, 2006, 2008]. Three linear relations (for separate time ranges determined due to the global changes of solar wind flux) between the solar wind proton density and speed were obtained from measurements of Ulysses during fast scans. Detailed description of the reconstruction is extensively presented in Sokól et al. [2012].

[6] Having the solar wind speed and density from Sokót et al. [2012], one can calculate the solar wind mass flux $\left(F_{s w}=n_{s w} V_{s w}\right)$ and the hydrogen charge exchange rate $\left(\beta_{e x}=n_{s w}\left|V_{s w}-V_{H}\right| \sigma_{e x}\left(\left|V_{s w}-V_{H}\right|\right) \approx n_{s w}\left|V_{s w}\right| \sigma_{e x}\left(\left|V_{s w}\right|\right)\right.$. Here we assumed that the speed of the hydrogen atom $V_{H}$ is much smaller than the solar wind speed $V_{s w}, \sigma_{e x}$ is the charge exchange cross section from Lindsay and Stebbings [2005]. In this paper we use charge exchange rates calculated by means of Sokół et al. [2012] results as input parameters 
to our 3-D time-dependent kinetic model of hydrogen distribution in the heliosphere [Katushkina and Izmodenov, 2012; Izmodenov et al., 2013]. Hydrogen distributions obtained from the model are used to calculate the intensities of backscattered solar Lyman-alpha radiation over the whole sky. Then these sky maps of the backscattered solar Lymanalpha intensities are compared with SOHO/SWAN measurements. Detailed description of the SWAN instrument can be found in Bertaux et al. [1995].

[7] The paper is organized as follows: in section 2, our kinetic model of hydrogen distribution in the heliosphere including the ionization rates is described; section 3 presents modelled full sky maps of the intensities of backscattered solar Lyman-alpha radiation and comparison with the SOHO/SWAN data; in discussion section 4, another possibility for deriving time and heliolatitudinal variations of the solar wind mass flux is considered.

\section{Advanced Kinetic Model of the Interstellar Hydrogen Distribution in the Heliosphere}

[8] Recently, Izmodenov et al. [2013]; and Katushkina and Izmodenov [2011, 2012] have developed an advanced kinetic model of interstellar hydrogen distribution in the heliosphere that allows to combine the simplicity of the hottype models [Lallement et al., 1985] with results of global self-consistent kinetic-gasdynamic models of the solar wind (SW) interaction with the Local Interstellar Medium (LISM) [Baranov and Malama, 1993; Izmodenov et al., 2009]. Generally, the model can be classified as a time-dependent 3-D version of the classical hot model with specific boundary conditions at $90 \mathrm{AU}$ obtained from results of the global kinetic-gasdynamic models of the SW/LISM interaction. In this section a brief description of the model is presented.

[9] Let us consider the interstellar hydrogen atoms $(\mathrm{H}$ atoms), which penetrate to the heliosphere from the LISM through the heliospheric interface region (i.e., region of the SW/LISM interaction). Interstellar atoms can be divided in two populations [Izmodenov et al., 2001]: primary and secondary. Primary interstellar atoms are originated in undisturbed interstellar medium and then penetrate to the heliosphere without being charge exchanged. Secondary interstellar atoms are created from the primary interstellar atoms during charge exchange with interstellar protons in a region of perturbed interstellar plasma (outside the heliopause). Inside the heliosphere, both primary and secondary interstellar atoms can be affected by charge exchange with the solar wind protons, and as a result, new heliospheric neutral hydrogen atoms will be created. But we do not consider such heliospheric atoms, because they do not influence the backscattered solar Lyman-alpha radiation due to small number densities and large velocities (see, however, Quemerais and Izmodenov [2002]).

[10] We are interested in the spatial and velocity distribution of the interstellar $\mathrm{H}$ atoms near the Sun, where they are measured by direct and indirect methods. Near the Sun interstellar $\mathrm{H}$ atoms are affected by solar gravitational attractive force $\mathbf{F}_{g}$ and solar radiation repulsive force $\mathbf{F}_{\text {rad }}$. These forces counteract each other and are proportional to $1 / r^{2}$, where $r$ is heliocentric distance. Let us define a dimensionless balanced parameter $\mu=\left|\mathbf{F}_{\text {rad }}\right| /\left|\mathbf{F}_{g}\right|$ that characterizes the ratio between solar gravitation and radiation forces. Then, the effective total force acting on each atom can be written in the following form:

$$
\begin{aligned}
\mathbf{F}\left(r, t, \lambda, w_{r}\right) & =\mathbf{F}_{r a d}\left(r, t, \lambda, w_{r}\right)+\mathbf{F}_{g}(r) \\
& =\left(1-\mu\left(t, \lambda, w_{r}\right)\right) \mathbf{F}_{g}=-m_{H} \frac{(1-\mu) G M_{s}}{r^{2}} \cdot \frac{\mathbf{r}}{r},
\end{aligned}
$$

here, $G$ is the gravitational constant, $M_{S}$ is the mass of the Sun, $m_{H}$ is the mass of $\mathrm{H}$ atom, and $\mathbf{r}$ is a radius-vector to a point. In general, $\mu$ depends on time $(t)$, heliolatitude $(\lambda)$ and radial component of atom's velocity $\left(w_{r}\right)$, because solar radiation force is caused by scattering of solar Lymanalpha photons by $\mathrm{H}$ atoms, and scattering process depends on radial atom's velocity.

[11] The kinetic equation for the velocity distribution function of $\mathrm{H}$ atoms can be written as follows:

$$
\begin{aligned}
\frac{\partial f(\mathbf{r}, \mathbf{w}, t)}{\partial t} & +\mathbf{w} \cdot \frac{\partial f(\mathbf{r}, \mathbf{w}, t)}{\partial \mathbf{r}} \\
& +\frac{\mathbf{F}\left(r, t, \lambda, w_{r}\right)}{m_{H}} \cdot \frac{\partial f(\mathbf{r}, \mathbf{w}, t)}{\partial \mathbf{w}}=-\beta(r, t, \lambda) \cdot f(\mathbf{r}, \mathbf{w}, t)
\end{aligned}
$$

[12] Here $f(\mathbf{r}, \mathbf{w}, t)$ is the velocity distribution function of the hydrogen atoms, $\mathbf{w}$ is the individual velocity of $\mathrm{H}$ atom, and $\beta$ is the total ionization rate.

[13] The right hand side of equation (1) represents losses of the atoms due to charge exchange $\left(H+H^{+} \leftrightarrow H^{+}+H\right)$ and photoionization $\left(H+h v=H^{+}+e\right)$ processes. Electron impact ionization is not taken into account due to its insignificance. The coefficient $\beta(r, t, \lambda)$ is the effective ionization rate: $\beta(r, t, \lambda)=\beta_{e x}(r, t, \lambda)+\beta_{p h}(r, t, \lambda)$, where $\beta_{e x}$ and $\beta_{p h}$ are rates of charge exchange and photoionization, respectively. It is assumed that these rates of ionization decrease with distance from the Sun as $\sim 1 / r^{2}$, since these values are proportional to the number density of the solar protons and flux of the solar photons, respectively. Therefore,

$$
\beta(r, t, \lambda)=\left(\beta_{e x, E}(t, \lambda)+\beta_{p h, E}(t, \lambda)\right)\left(\frac{r_{E}}{r}\right)^{2}=\beta_{E}(t, \lambda)\left(\frac{r_{E}}{r}\right)^{2},
$$

where $r_{E}=1 \mathrm{AU}$ is the distance from the Earth to the Sun. The subscript $E$ indicates the values taken are at $1 \mathrm{AU}$.

[14] Functions $\mu\left(t, \lambda, w_{r}\right), \beta_{e x, E}(t, \lambda)$, and $\beta_{p h, E}(t, \lambda)$ are the input parameters of the model, and we take them from the experimental data.

[15] Detailed description of the function $\mu\left(t, \lambda, w_{r}\right)$ is presented in Izmodenov et al. [2013].

[16] The charge exchange rate at $1 \mathrm{AU}$ as a function of time and heliolatitude $\left(\beta_{e x, E}(t, \lambda)\right)$ is taken from the results of Sokót et al. [2012]. For the purposes of this paper, time-resolution of the solar wind parameters from results of Sokól et al. [2012] was increased from 1 year to one Carrington rotation by simple linear interpolation in time. Then the charge exchange rate can be calculated as follows: $\beta_{e x, E}=n_{p, E} V_{s w, E} \sigma_{e x}\left(V_{s w, E}\right)$, where, $n_{p, E}$ is the solar wind proton number density at $1 \mathrm{AU}, V_{s w, E}$ is the solar wind speed at $1 \mathrm{AU}, \sigma_{e x}\left(V_{s w}\right)$ is a charge exchange cross-section from Lindsay and Stebbings [2005]. Figure 1a presents the charge exchange rate at $1 \mathrm{AU}$ based on the results of Sokót et al. [2012].

[17] To calculate the photoionization rate $\left(\beta_{p h, E}\right)$ at $1 \mathrm{AU}$ as a function of time in the ecliptic plane, we use SOLAR2000 database (http:/www.spacewx.com/ solar2000.html; the Solar Irradiance Platform (SIP), formerly the SOLAR2000 irradiance specification tool [Tobiska 

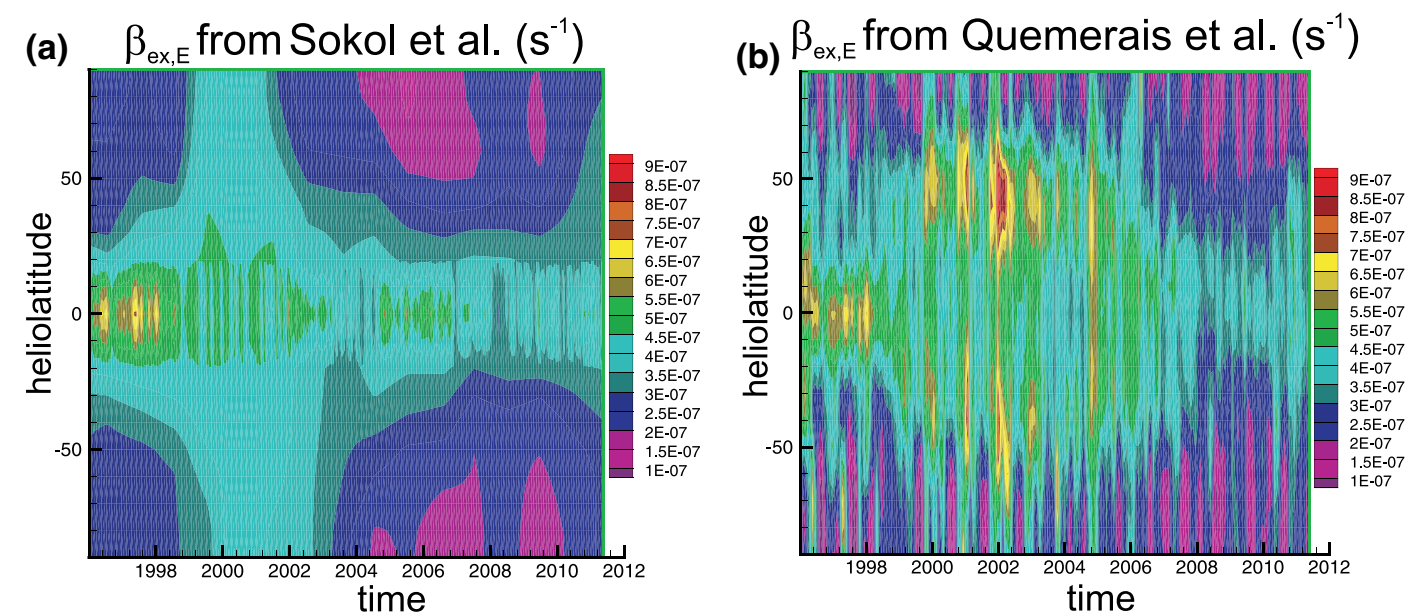

Figure 1. The charge exchange ionization rates at $1 \mathrm{AU}$ as functions of time and heliolatitude for two data sets. (a) Results of [Sokót et al., 2012] based on different measurements (interplanetary scintillations, Ulysses, OMNI-2). (b) Results of [Quemerais et al., 2006] based on the analysis of Lyman-alpha intensities from $\mathrm{SOHO} / \mathrm{SWAN}$ (see section 4).

et al., 2000], produces the variable, full solar spectrum in assorted spectral formats for historical, nowcast, and forecast applications). The time resolution of these data is 1 day. In the calculations we neglect the angle between the solar equatorial and ecliptic planes, i.e., we assume that from SOLAR2000, we get $\beta_{p h, E}(t, \lambda=0)$. Magnitudes of $\beta_{p h, E}(t, \lambda=0)$ were adjusted from the Earth orbit to $1 \mathrm{AU}$ and then were averaged over one Carrington rotation. We assume that heliolatitudinal dependence of $\beta_{p h, E}(t, \lambda)$ is the same as for charge exchange rate, i.e., $\beta_{p h, E}(t, \lambda)=\beta_{p h, E}(t, 0)$. $\beta_{e x, E}(t, \lambda) / \beta_{e x, E}(t, 0)$.

[18] For modelling of the hydrogen distribution, we solve the seven-dimensional (three coordinates in space, three components of velocity, and time) kinetic equation (1) for the velocity distribution function. At the outer boundary (Sun centered sphere with radius 90 AU), we use nonMaxwellian boundary conditions for the distribution function that takes into account changes of hydrogen distribution during atom's motion through the heliospheric interface region [Izmodenov et al., 2001; Katushkina and Izmodenov, 2010, 2012; Izmodenov et al., 2013]. These changes are caused by the charge exchange process between the interstellar hydrogen atoms and protons. In this paper we use the boundary conditions in the form of 3-D normal distribution (i.e., we take into account all zero, first, and second moments of velocity distribution function at $90 \mathrm{AU}$, see Katushkina and Izmodenov [2010]) separately for the primary and secondary interstellar atoms. If it is not specified otherwise, parameters of the boundary distributions were taken from results of stationary axisymmetric self-consistent kineticgasdynamic model of the heliospheric interface [Baranov and Malama, 1993] with the following parameters of the LISM: the number densities of protons and neutral hydrogen atoms are $n_{\mathrm{p}, \mathrm{LISM}}=0.06 \mathrm{~cm}^{-3}, n_{\mathrm{H}, \mathrm{LISM}}=0.18 \mathrm{~cm}^{-3}$, respectively; the relative LISM/solar wind velocity is $V_{\text {LISM }}=$ $26.4 \mathrm{~km} / \mathrm{s}$, and direction opposite to $\mathbf{V}_{\text {LISM }}$ (i.e., the upwind direction) has $255.4^{\circ}$ of ecliptic longitude and $5.2^{\circ}$ of ecliptic latitude [Lallement et al., 2010], and the interstellar temperature is $T_{\text {LISM }}=6519 \mathrm{~K}$.

\section{Results: Lyman-Alpha Intensity Maps, Comparison With SOHO/SWAN Data}

[19] Based on the hydrogen distribution obtained as described above, we calculated full sky maps of the backscattered solar Lyman-alpha intensities. To do this we solve the radiative transfer equation for the backscattered solar Lyman-alpha spectra in the frame of simplified self-absorption approach [see Quemerais and Izmodenov, 2002]. In this approximation, only singly scattered photons are taken into account and extinction along the line of sight between the scattering point and the observer is included. Multiple scattering (neglected here) leads to increasing the intensities for all lines of sight, but it does not change the position of the maximum and minimum intensities. In this paper we will analyze only the spatial distribution of the backscattered solar Lyman-alpha intensity, and we do not consider the absolute values of the intensities. So, self-absorption approximation is sufficient for our purposes.

[20] Figures 2 and 3 present the results of calculations for the minimum (1997 and 2009) and maximum (2002-2005) of solar activity. Lyman-alpha intensity maps were calculated as they would be seen by SOHO spacecraft, i.e., the positions of observer for these maps correspond to the actual SOHO orbital positions. We consider the maps obtained on 1 June each year, when the Earth is located near the upwind direction.

[21] Now let us focus on the first and second columns in Figures 2 and 3 (the third and fourth columns will be considered later in section 4). First columns in Figures 2 and 3 present normalized SOHO/SWAN Lyman-alpha intensities as functions of ecliptic longitude and latitude. Second columns present the normalized maps obtained theoretically in our model with the charge exchange rate from Sokól et al. [2012]. For each map, all intensities are normalized to the intensity in one chosen direction (with ecliptic latitude $80.5^{\circ}$ and ecliptic longitude $120.5^{\circ}$ ) located in the background. We consider normalized intensities here, because in this paper 

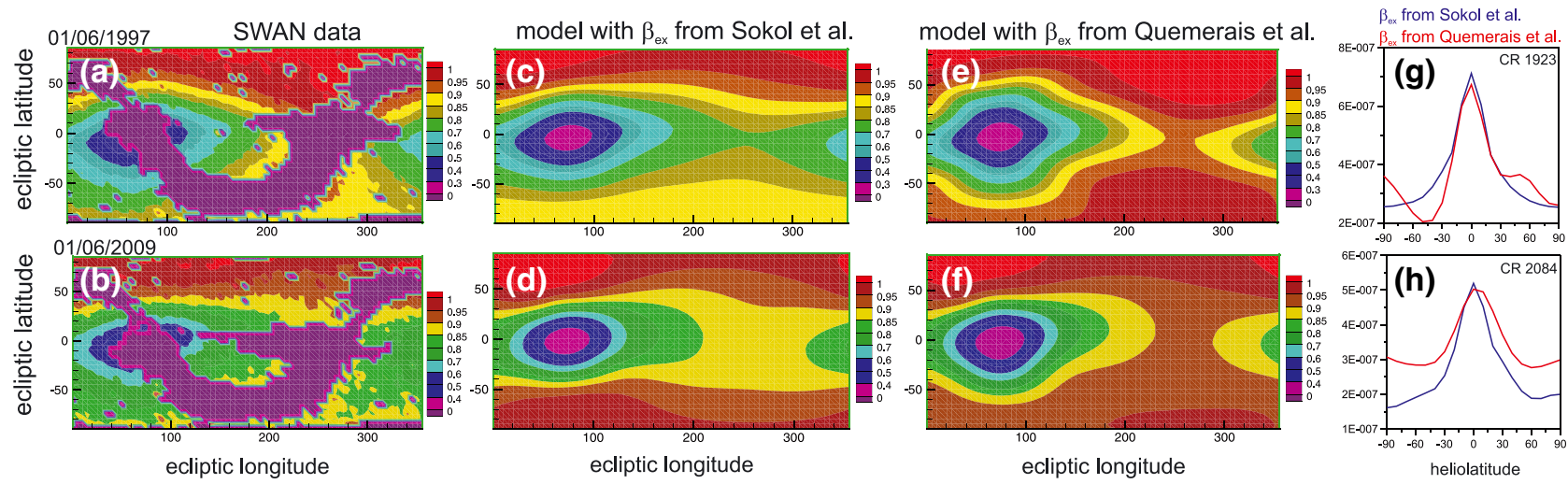

Figure 2. Results of calculations for period of the solar minimum (1997 and 2009). (a-f) Full sky maps of the backscattered solar Lyman-alpha intensities in the ecliptic coordinates. For each map, all intensities are normalized at corresponding intensity in the chosen direction (with ecliptic latitude $80.5^{\circ}$ and ecliptic longitude $120.5^{\circ}$ ). Figures $2 \mathrm{a}$ and $2 \mathrm{~b}$ show the SOHO/SWAN data. Note that the ribbon with zero intensities over the whole maps in the SWAN data is caused by the shadow of the spacecraft. Figures $2 \mathrm{c}$ and $2 \mathrm{~d}$ show the results of the numerical model with the charge exchange ionization rate based on Sokót et al. [2012]. Figures 2e and 2f show the results of numerical model with charge exchange ionization rate based on Quemerais et al. [2006]. (g and h) The charge exchange ionization rates as functions of heliolatitude for two data sets. CR is a number of Carrington rotation.

we are interested in the qualitative effects and, hence, we are going to study only the shapes of the Lyman-alpha intensity maps. Absolute intensities will be studied by us separately in a future paper. We can see that during the solar minima, our numerical results are very close to the SWAN data. At least the shapes of the intensity maps coincide very well (compare Figures $2 \mathrm{a}$ and $2 \mathrm{c}, 2 \mathrm{~b}$ and $2 \mathrm{~d}$ ). But at the solar maximum, significant differences between model results and SWAN data appear (compare first and second columns in Figure 3). SWAN data shows that in 2002-2005, Lymanalpha intensity has maximum near the upwind direction. While in the model results, maximum of intensities is located closer to the north ecliptic pole. And in general, during the solar maximum, theoretical and experimental maps look qualitatively different.

[22] In order to understand the reason of the differences, we study which physical process has a major influence on the spatial pattern of Lyman-alpha intensity maps. The distribution of interstellar hydrogen near the Sun is determined by three concurrent factors. The first factor is changes of hydrogen distribution in the heliospheric interface region due to charge exchange with the interstellar protons. For example, as the result of the charge exchange, new secondary interstellar atoms are created, and their properties are different as compared with the primary interstellar atoms. This factor is taken into account in our model through the non-Maxwellian boundary conditions at $90 \mathrm{AU}$. Second factor is the curvature of $\mathrm{H}$ atoms trajectories near the Sun due to the influence of the solar gravitation and radiation pressure. Quantitatively, this factor is represented by $\mu$-value in the model. And the third factor is the charge exchange (characterized by charge exchange rate $\beta_{e x, E}$ ) affecting $\mathrm{H}$ atoms in the heliosphere. We do not mention here the photoionization rate, because it is much less than the charge exchange rate and can not influence the results considerably. We performed additional test calculations to check the role of the boundary conditions and parameter $\mu$.
[23] The first test map was calculated for 1 June 2005, when the differences between SWAN data and model are mostly pronounced (see Figures $3 \mathrm{~d}$ and $3 \mathrm{~h}$ ). This test illustrates the influence of the boundary conditions in our model of hydrogen distribution. A 3-D normal distribution [see, Katushkina and Izmodenov, 2010] has been employed as the boundary condition. The parameters of this distribution were calculated in the frame of 3-D stationary kinetic-MHD model of the solar wind interaction with the local interstellar medium [Izmodenov et al., 2005, 2009]. LISM parameters (i.e., velocity vector and number density) in the model were chosen to be reasonable, but essentially different from those of previously considered axysimmetric model. Namely, the number densities of protons and neutral hydrogen atoms are $n_{\mathrm{p}, \text { LISM }}=0.04 \mathrm{~cm}^{-3}$ and $n_{\mathrm{H}, \mathrm{LISM}}=0.14 \mathrm{~cm}^{-3}$, respectively; the relative LISM/solar wind velocity is $V_{\text {LISM }}=$ $23.2 \mathrm{~km} / \mathrm{s}$, and direction opposite to $\mathbf{V}_{L I S M}$, i.e., the upwind direction has $259.0^{\circ}$ of ecliptic longitude and $4.98^{\circ}$ of ecliptic latitude, interstellar temperature is the same as previous $T_{\text {LISM }}=6519 \mathrm{~K}$, interstellar magnetic field $B_{\text {LISM }}=4.4 \mu G$ is taken into account and angle between $\mathbf{V}_{L I S M}$ and $\mathbf{B}_{L I S M}$ is $20^{\circ}$. The magnitude and direction of $\mathbf{V}_{\text {LISM }}$ are based on analysis of IBEX data and were taken from McComas et al. [2012]. Corresponding hydrogen distribution at $90 \mathrm{AU}$ adopted as the boundary condition for this test calculation is non-axisymmetric (3-D) due to the influence of the interstellar magnetic field. However, despite some asymmetry and quantitative differences, $\mathrm{H}$ atom parameters at $90 \mathrm{AU}$ in 3-D model are qualitatively similar to those in the axisymmetric model, which are described in Figure 3 from Katushkina and Izmodenov [2010]. To study details of the heliospheric interface effects on the velocity distribution function is out of the scope of this paper. We also do not discuss here possible effects of the heliospheric magnetic field (HMF) on the SW/LISM interaction, because HMF strongly changes the plasma flow, but barely affect the filtration of the interstellar atoms as it will be shown in a future paper. Results of the test 

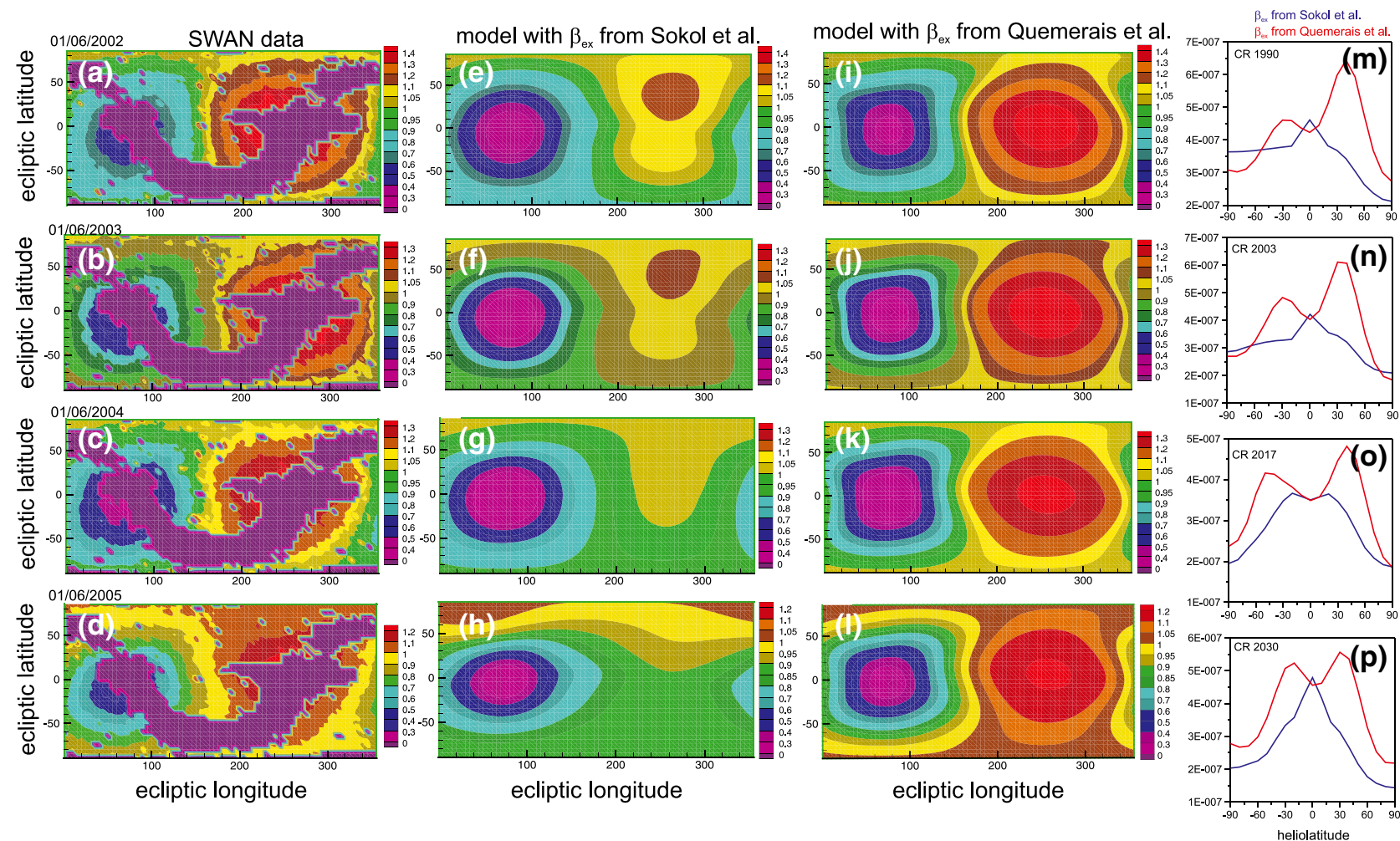

Figure 3. Results of calculations for period of the solar maximum (2002-2005). (a-1) Full sky maps of the backscattered solar Lyman-alpha intensities in the ecliptic coordinates. For each map, all intensities are normalized at corresponding intensity in the chosen direction (with ecliptic latitude $80.5^{\circ}$ and ecliptic longitude $120.5^{\circ}$ ). Figures $3 \mathrm{a}-3 \mathrm{~d}$ show the SOHO/SWAN data. Note that the ribbon with zero intensities over the whole maps in the SWAN data is caused by the shadow of the spacecraft. Figures $3 \mathrm{e}-3 \mathrm{~h}$ show the results of numerical model with The charge exchange ionization rate based on Sokót et al. [2012]. Figures 3i-31 show the results of numerical model with charge exchange ionization rate based on Quemerais et al. [2006]. (m-p) The charge exchange ionization rates as function of heliolatitude for two data sets. CR is a number of Carrington rotation.

are presented in Figure 4a. It is seen that the obtained map is very close to the map shown in Figure $3 \mathrm{~h}$. We conclude, therefore, that physically reasonable changes in the boundary conditions do not change the shape of the backscattered solar Lyman-alpha intensity maps.

[24] The parameter $\mu$ varies from approximately 0.95 in the solar minimum to 1.6 in the solar maximum [see Izmodenov et al., 2013]. In order to illustrate the influence of $\mu$-value, we performed the second test. The calculations were performed for 1 June 2002, since 2002 corresponds to a peak of the solar maximum, and $\mu$-value in that time was the largest. Lyman-alpha intensity map was calculated in the frame of our model with standard boundary conditions under assumption of constant $\mu=0.97$. This magnitude of $\mu$ corresponds to the solar minimum conditions and is very different from real value of $\mu$ in 2002. Results of this test are presented in Figure $4 \mathrm{~b}$. It is seen that strong decrease of $\mu$-value produces small changes in the Lyman-alpha intensity map (compare Figure 4a and Figure 3e), but an agreement with the SWAN data becomes even worse. So we have shown that neither large $\mu$-values, nor small $\mu$-values can not help to get an agreement between the model results and the SWAN data during the solar maximum.

[25] Thus, we can conclude, that only charge exchange rate $\beta_{e x, E}(t, \lambda)$, may be responsible for the shape of Lyman- alpha intensity maps. Therefore, significant differences between model results and SWAN data in 2002-2005 show that charge exchange rate from Sokót et al. [2012] is not consistent with Lyman-alpha intensities measured by SOHO/SWAN during the period of solar maximum activity. While during the solar minimum activity, the charge exchange rate allows to obtain a qualitative agreement in the Lyman-alpha intensities with the SWAN data.

\section{Conclusion and Discussion}

[26] It is concluded in this paper that the data set of heliolatitudinal and time variations of the solar wind parameters obtained by Sokól et al. [2012] disagrees with SOHO/SWAN data of the backscattered solar Lyman-alpha intensities for the solar maximum (2002-2005).

[27] One possible reason for the disagreement could be in the assumption of linear correlation between the solar wind speed and density made by Sokót et al. [2012]. As it is seen from Figure 17 of Sokót et al. [2012], this assumption is appropriate for the solar minimum, but it does not work for the solar maximum, i.e., exactly when the disagreement with SOHO/SWAN data was established. The possible incorrectness in the correlation may lead to inaccuracies in determination of the solar wind number 

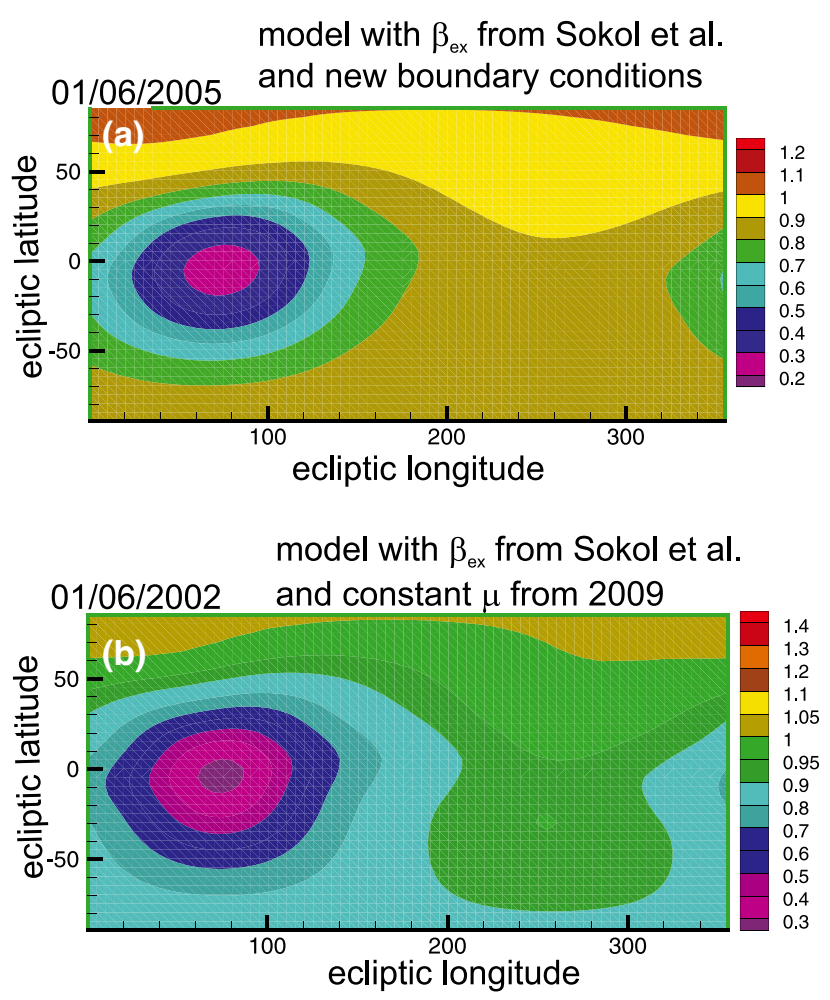

Figure 4. Results of test calculations. (a) Results of the first test. This is Lyman-alpha intensity map obtained in 2005 with different boundary conditions (see text for details). (b) Results of the second test. This is Lyman-alpha intensity map obtained in 2002 with broken value of $\mu$ (taken from 2009).

density and then corresponding inaccuracies in the charge exchange rate.

[28] The question is, what are other possibilities to determine the heliolatitudinal and time variations of the solar wind parameters. One way of indirect diagnostic of 3-D solar wind structure is based on observations of heliospheric EUV emission. For example, Gruntman [2001] and Gruntman et al. [2006] studied theoretically a distribution of EUV emission in the $30.4-\mathrm{nm}$ line produced in the charge exchange collisions between the solar wind alpha particles and atomic hydrogen in the heliosphere. It was shown in the cited papers of Gruntman that full-sky images at 30.4$\mathrm{nm}$ "reveal the three-dimensional flow properties of the solar wind, including the flow in the regions over the Sun's poles and the variability of the global solar wind properties during the 11-year solar cycle." In the paper by Pryor et al. [2003], heliolatitudinal anisotropy of the hydrogen charge exchange rates are studied based on analysis of Ulysses/GAS Lymanalpha intensities. It was found in this work that during the solar minimum time period, Lyman-alpha brightness is minimum near the upwind direction, while during the solar maximum, Lyman-alpha brightness is on contrary maximum near the upwind direction. These results are consistent with SWAN data presented in the current paper. Authors of the paper Pryor et al. [2003] concluded that during the solar minimum, when heliospheric current sheet (HCS) tilt was small, charge exchange rate is enhanced at low latitudes. But in the solar maximum, when HCS is more inclined, charge exchange rate became more latitudinally isotropic.
[29] Currently the most developed way to study the solar wind through the EUV emission is to use the data from SWAN (Solar Wind ANisotropy) instrument on board the SOHO spacecraft. This instrument is specifically devoted to study the large scale structure and heliolatitudinal variations of the solar wind by measurements of backscattered solar Lyman-alpha radiation. Detail description of the SWAN mission can be found in Bertaux et al. [1995, 1997]. Latitudinal and time variations of the total hydrogen ionization rate at $1 \mathrm{AU}$ can be obtained as a solution of inverse problem from analysis of backscattered Lyman-alpha maps based on a model of interstellar $\mathrm{H}$ atoms in the heliosphere. Detailed description of the method is presented in Quemerais et al. [2006], and recent results are presented in Lallement et al. [2010]. In this method a 3-D quasi-stationary kinetic "hot" model for the hydrogen distribution [Lallement et al., 1985] was used to calculate the hydrogen parameters and then theoretical Lyman-alpha intensity maps. In the model the total ionization rate [i.e., sum of the charge exchange rate (main component) and photoionization rate] of the interstellar $\mathrm{H}$ atoms at $1 \mathrm{AU}$ was assumed to be a free function of heliolatitude. Then this function was varied in order to get the best fit with a corresponding $\mathrm{SOHO} / \mathrm{SWAN}$ intensity map. As a result, the total ionization rate at $1 \mathrm{AU}\left(\beta_{\text {tot,E,SWAN }}(t, \lambda)\right)$ as a function of heliolatitude and time (from 1996 up to now) was obtained.

[30] In order to get charge exchange rate $\beta_{e x, E}(t, \lambda)$ and photoionization rate $\beta_{p h, E}(t, \lambda)$ separately from SWAN results, we can apply the following procedure. In the ecliptic plane we know both ionization rates as functions of time from OMNI-2 and SOLAR2000 databases. Then, if we assume that heliolatitudinal variations of the photoionization rate are the same as for the charge exchange rate, we can obtain the following:

$$
\begin{aligned}
& \beta_{e x, E}(t, \lambda)=\beta_{e x, E, O M N I}(t, 0) \cdot \frac{\beta_{t o t, E, S W A N}(t, \lambda)}{\beta_{t o t, E, S W A N}(t, 0)} \\
& \beta_{p h, E}(t, \lambda)=\beta_{p h, E, S O L A R 2000}(t, 0) \cdot \frac{\beta_{t o t, E, S W A N}(t, \lambda)}{\beta_{t o t, E, S W A N}(t, 0)} .
\end{aligned}
$$

Here we assume that heliolatitude is approximately equal to zero at the ecliptic plane. The charge exchange rate is a product of the solar wind proton mass flux and the energy dependent charge exchange cross-section. It means that if we know the charge exchange rate from SWAN and solar wind velocity from some other data (e.g., from Sokót et al. [2012] based on interplanetary scintillations), we can obtain the solar wind proton number density as a function of time and heliolatitude. Namely

$$
n_{p, E}(t, \lambda)=\frac{\beta_{e x, E}(t, \lambda)}{V_{p, E}(t, \lambda) \cdot \sigma\left(\left|V_{p, E}\right|\right)},
$$

here, $V_{p, E}(t, \lambda)$ is the solar wind velocity from results of Sokót et al. [2012] based on the IPS data, $\sigma$ is the charge exchange cross-section.

[31] The charge exchange rate at $1 \mathrm{AU}$ obtained from the SWAN data is presented in Figure $1 \mathrm{~b}$. It is seen that there are considerable (even qualitative) differences from the charge exchange rate of Sokót et al. [2012]. For example, charge exchange rate obtained from SWAN data has two characteristic maxima at about $\pm 40^{\circ}-50^{\circ}$ of heliolatitude for the solar maximum time-period (2000-2005), 

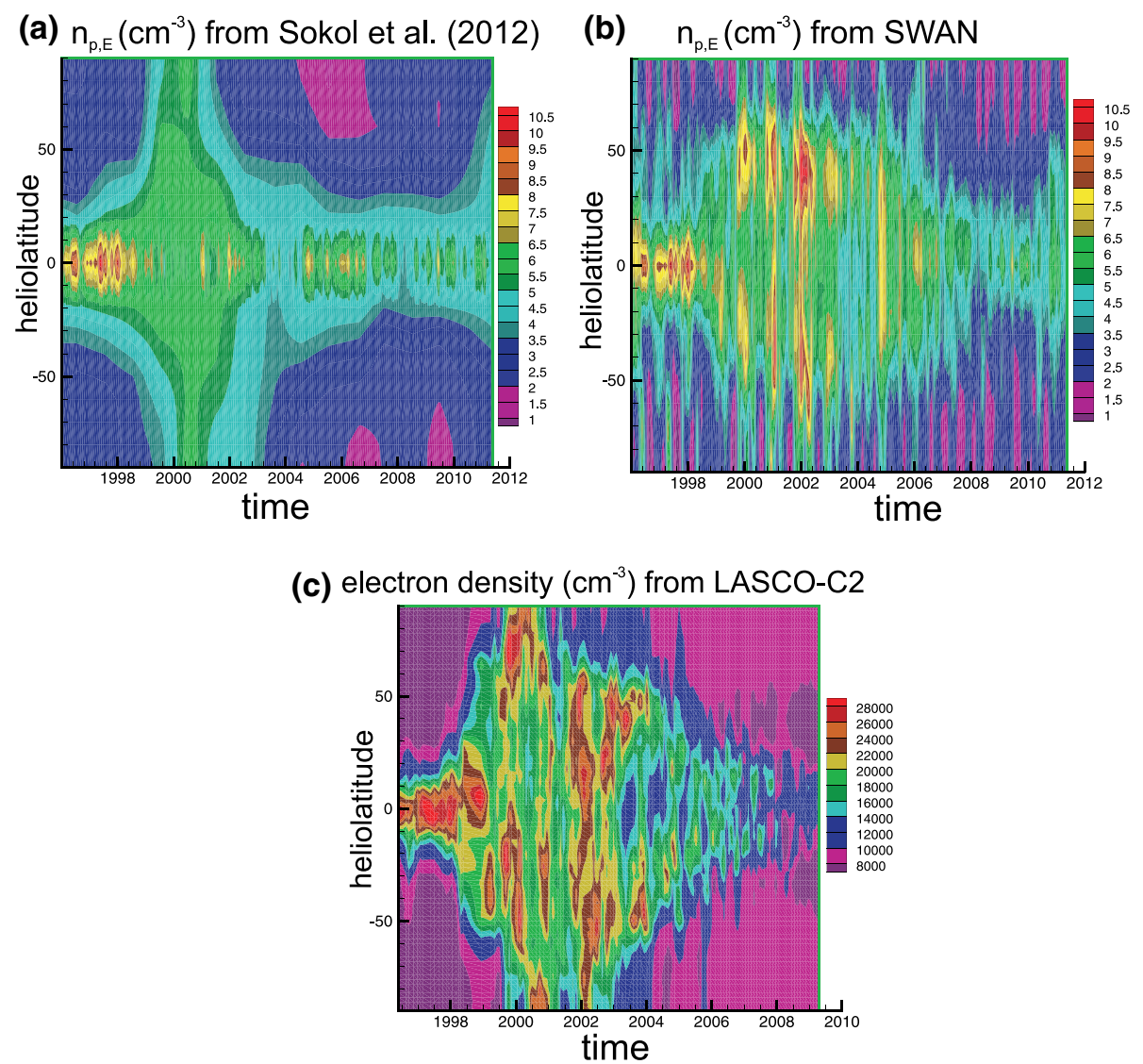

Figure 5. (a) Solar wind proton number density at $1 \mathrm{AU}$ as a function of time and heliolatitude derived by Sokót et al. [2012] from the Interplanetary Scintillations data, OMNI-2 database in the ecliptic plane, and Ulysses data out of the ecliptic plane; (b) solar wind proton number density at 1 AU derived from the charge exchange rate (obtained from the SWAN data) and solar wind speed from the results of Sokót et al. [2012]; (c) coronal electron number density at six solar radii obtained from the white light brightness measured by SOHO/LASCO-C2 [Quemerais and Lamy, 2002].

while this feature is absent in the first data set based on Sokót et al. [2012] results.

[32] Figure 5 presents the solar wind proton number density as a function of time and heliolatitude obtained from the SWAN data (Figure 5b) by the described above procedure, and from the results of Sokót et al. [2012] (Figure 5a) based on IPS data, OMNI-2 and Ulysses data. It is seen that qualitative behavior of the proton number density is the same as that for the charge exchange rate. There are also two maxima at middle heliolatitudes during time period from 2000 to 2005 for the number density derived from SWAN. And these maxima are absent in the results of Sokót et al. [2012].

[33] It is interesting to compare both charge exchange rates with the charge exchange rate calculated from the in situ measurements of the solar wind number density and speed by Ulysses spacecraft. For this comparison the solar wind speed and density measured by Ulysses/SWOOPS (ftp://nssdcftp.gsfc.nasa.gov/spacecraft $t_{d}$ ata/ulysses/plasmal swoops/ion/hour/) were adjusted to $1 \mathrm{AU}$ and averaged over the Sun's rotation. Figure 6 presents results of the comparison for 1996-2009 time period. Figures $6 \mathrm{~b}$ and $6 \mathrm{c}$ show the comparison only during Ulysses's fast scans (2001-2002 and 2007-2008). It is seen from Figure 6 that in general both data sets represent Ulysses's data well, although there are some local differences. Note, that differences in the charge exchange ionization rate between results of the SWAN analysis and direct measurements of Ulysses are most probably connected with different kinds of diagnostics. Ulysses has performed direct measurements of the solar wind parameters along its trajectory. But from the analysis of the Lymanalpha intensities measured by SWAN, one can obtain the ionization rates averaged over heliolongitude. Therefore, some local differences between two methods should exist, and it is not surprising. However, from Figure $6 \mathrm{a}$, it is seen that, in general, agreement between Ulysses and SWAN is sufficiently good, except for some local differences during short time periods.

[34] In order to study how our results of the Lymanalpha intensity maps are sensitive to the charge exchange rate, we performed our 3-D time-dependent calculations as described in the previous section, but using charge exchange rate from SWAN analysis. Results of the calculations are presented in Figures $2 \mathrm{e}-2 \mathrm{f}$ and $3 \mathrm{i}-3 \mathrm{l}$ ). We see from the figures that these model results have very good agreement with the data for both solar minimum and solar maximum conditions. From this fact, we can make two conclusions. First, the spatial pattern of the backscattered Lyman-alpha maps (or shape of normalized Lyman-alpha intensity maps) 

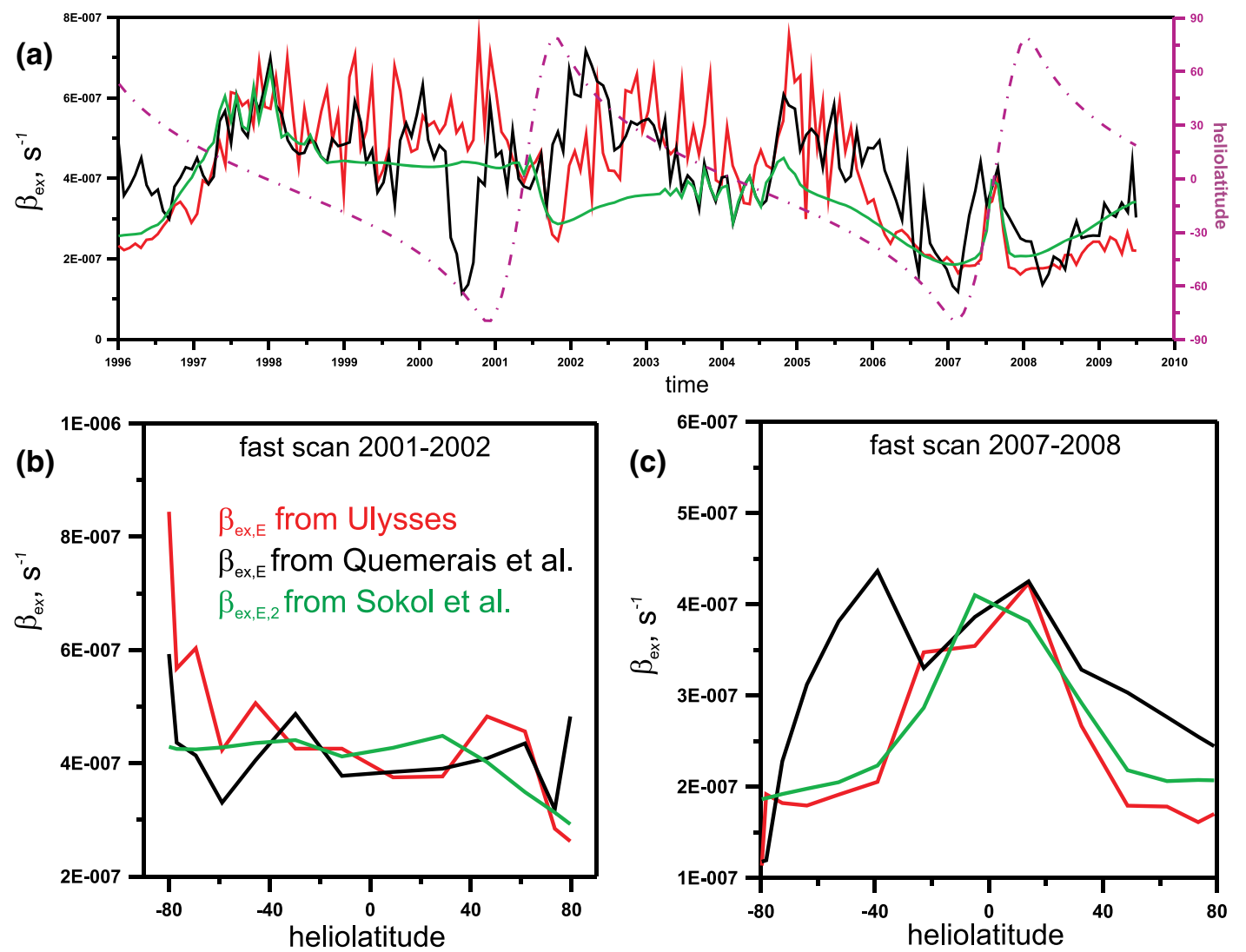

Figure 6. (a) The charge exchange rates at 1 AU as functions of time along the Ulysses's trajectory. In all panels, red (or dark-grey in printed version) curves correspond to the data from Ulysses measurements (adjusted to $1 \mathrm{AU}$ and averaged over one rotation of the Sun); green (or light-grey in printed version) curves correspond to the charge exchange rates from Sokót et al. [2012], black curves correspond to the charge exchange from analysis of the SWAN data; purple dash-dotted curve represents heliolatitude of Ulysses during its motion. (b) The charge exchange rates as functions of heliolatitude along Ulysses's trajectory during its second fast scan in 2001-2002. (c) The charge exchange rates as functions of heliolatitude along Ulysses's trajectory during its third fast scan in 2007-2008.

is mostly determined by local heliolatitudinal anisotropy of the charge exchange rate. Changes only in the charge exchange rate lead to qualitatively different Lyman-alpha intensity maps. Second, if one is interested only in heliolatitudinal variations of the ionization rates (and is not interested in its absolute values) the quasi-stationary approach used by Quemerais et al. [2006] works properly. Because, although in the inversion procedure for obtaining of the total ionization rates from SWAN a simplified quasi-stationary hot model was used, our calculations in the frame of timedependent model allow to obtain similar Lyman-alpha intensity maps. It means, that time-depended effects are not important for what we consider here.

[35] Figures $2 \mathrm{~g}-2 \mathrm{~h}$ and Figures $3 \mathrm{~m}-3 \mathrm{p}$ show the charge exchange rates for the chosen moments of time as a function of heliolatitude derived from Sokól et al. [2012] and from Quemerais et al. [2006]. It it seen that during the solar minimum conditions (Figure 2), heliolatitudinal dependence of the charge exchange rates are quite similar for two data sets, but during solar maximum conditions (Figure 3), the charge exchange rates are qualitatively different. SOHO/SWAN Lyman-alpha intensities in 2002-2005 show that the charge exchange rate should have two maxima at middle latitudes $\pm 40^{\circ}-50^{\circ}$, while results of Sokół et al. have one maximum of $\beta_{e x, E}$ at zero latitude.

[36] Note that there is an additional experimental indirect confirmation of the existence of these two maxima of the solar wind mass flux. This is a heliolatitudinal distribution of Thomson-scattered brightness observed at 2.5 solar radii by SOHO LASCO-C2 [see Figure 5 in Manoharan, 2012] and corresponding electron density at six solar radii derived from LASCO-C2 images [see Figure 3 in Lallement et al., 2010; Quemerais and Lamy, 2002]. Figure 5c presents number density of the solar wind electrons at six solar radii, which was obtained from the LASCO-C2 data. Detailed description of the method of transformation from the measured white light brightness to the electron number density is presented in Quemerais and Lamy [2002], and some results of the analysis can be found in Quemerais et al. [2007]. It is seen from the comparison of Figures $5 \mathrm{a}$ and $5 \mathrm{~b}$ with $5 \mathrm{c}$ that temporal and heliolatitudinal variations of the coronal electron density show similar behavior (with two maxima in 2000-2005) as we obtained for the proton number density at 1 AU from the SWAN data. Of course, electron density at six solar radii (in solar corona) could not be compared directly with the proton number density at $1 \mathrm{AU}$, some differences 
may exist. But there is a good qualitative agreement, which confirms independently that some physical processes on the Sun lead to formation of this two maxima structure in number density of both protons and electrons during the solar maximum time period. However, physical reasons of these maxima are not clear so far and should be studied and explained in future works. The location of these maxima is quite possibly linked to the distribution of active regions on the solar surface and its evolution with the 11 year solar cycle.

[37] Knowledge of time and heliolatitudinal variations of the solar wind parameters is essential for the global modeling of the solar wind interaction with the local interstellar medium. We suppose that large differences in the solar wind mass flux between two available data sets discussed here would lead to significant differences in global dynamics of the heliosphere. Therefore, comparison of the global model results with the outer heliosphere data (e.g., solar wind parameters measured in the heliosheath by Voyager 1 and 2) would be an additional test for the solar wind mass flux discussed here. This is the subject for future studies.

[38] Acknowledgments. We acknowledge the SOHO mission. SOHO is a mission of cooperation between ESA and NASA. SWAN was developed in cooperation between LATMOS, France (lead funding agency CNES) and FMI, Helsinki, Finland. The authors thank the LASCO-C2 PI for providing the LASCO-C2 white light polarized brightness maps. Also, we acknowledge the OMNI-2 data collection (ftp://nssdcftp.gsfc.nasa.gov/spacecraft data/omni/). The authors would like to thank ISSI for their support of the working group. The calculations were performed by using the supercomputers of the Lomonosov Moscow State University ("Lomonosov" and "Chebyshev"). O.K. and V.I. are partially supported by grant of Russian Ministry of Education and Science (8413), RFBR grant 13-01-00265 and Programm 22 of the Russian Academy of Sciences. J.M.S. is supported by the grant NS-1260-11-09 from the Polish Ministry for Science and Higher Education. We thank M. Opher and P.K. Manoharan for careful reviewing of the manuscript.

[39] Philippa Browning thanks the reviewers for their assistance in evaluating this paper.

\section{References}

Baranov, V. B., and Y. G. Malama (1993), Model of the solar wind interaction with the local interstellar medium-Numerical solution of self-consistent problem, J. Geophys. Res., 98, 15,157-15,163.

Bertaux, J. L., et al. (1995), SWAN: A study of solar wind anisotropy on SOHO with Lyman alpha sky mapping, Sol. Phys., 162, 403-439.

Bertaux, J. L., et al. (1997), First results from SWAN Lyman alpha solar wind mapper on SOHO, Sol. Phys., 175, 737-770.

Gruntman, M. (2001), Imaging the three-dimensional solar wind, J. Geophys. Res., 106, 8205-8216.

Gruntman, M., V. Izmodenov, and V. Pizzo (2006), Imaging the global solar wind flow in EUV, J. Geophys. Res., 111, A04216, doi:10.1029/2005JA011530.

Izmodenov, V. V., M. Gruntman, and Y. G. Malama (2001), Interstellar hydrogen atom distribution function in the outer heliosphere, J. Geophys. Res., 106, 10,681-10,689.

Izmodenov, V., D. Alexashov, and A. Myasnikov (2005), Direction of the interstellar $\mathrm{H}$ atom inflow in the heliosphere: Role of the interstellar magnetic field, Astron. Astrophys., 437, L35-L38.

Izmodenov, V. V., et al. (2009), Kinetic-gasdynamic modeling of the heliospheric interface, Space Sci. Rev., 146, 329-351.
Izmodenov, V. V., et al. (2013), Distribution of interstellar hydrogen atoms in the heliosphere and backscattered solar Lyman-alpha, in CrossCalibration of Far UV Spectra of Solar System Objects and the Heliosphere, ISSI Sci. Rep. Ser., vol. 13, edited by E. Quemerais et al., chap. 1, pp. 7-65, Springer, New York, doi:10.1007/978-1-4614-6384-92.

Jackson, B. V., et al. (1998), Heliospheric tomography using interplanetary scintillation observations. 1. Combined Nagoya and Cambridge data, $J$. Geophys. Res., 103, 12,049-12,067.

Katushkina, O. A., and V. V. Izmodenov (2010), Effect of the heliospheric interface on the distribution of interstellar hydrogen atom inside the heliosphere, Astron. Lett., 36, 297-306.

Katushkina, O. A., and V. V. Izmodenov (2011), Spectral properties of backscattered solar Lyman-alpha radiation in the heliosphere: A search for heliospheric boundary effects, Adv. Space Res., 48, 1967-1979.

Katushkina, O. A., and V. V. Izmodenov (2012), The influence of effects of the heliospheric interface on parameters of backscattered solar Lymanalpha radiation measured at the Earth's orbit, Cosmic Res., 50, 141-151.

King, J. H., and N. E. Papitashvili (2005), Solar wind spatial scales in and comparisons of hourly Wind and ACE plasma and magnetic field data, $J$. Geophys. Res., 110, A02104, doi:10.1029/2004JA010649.

Lallement, R., J. L. Bertaux, and F. Dalaudier (1985), Interplanetary Lyman-alpha spectral profiles and intensities for both repulsive and attractive solar force fields: Predicted absorption pattern by a hydrogen cell, Astron. Astrophys., 150, 21-32.

Lallement, R., et al. (2010), The interstellar H flow: Updated analysis of SOHO/SWAN data, AIP Conf. Proc., 1216, 555-558.

Lindsay, B. G., and R. F. Stebbings (2005), Charge transfer cross sections for energetic neutral atom data analysis, J. Geophys. Res., 110, A12213, doi:10.1029/2005JA011298.

Manoharan, P. K. (2012), Three-dimensional evolution of solar wind during solar cycles 22-24, Astrophys. J., 751, 128.

McComas, D. J., et al. (2000), Solar wind observations over Ulysses' first full polar orbit, J. Geophys. Res., 105, 10,419-10,434.

McComas, D. J., et al. (2003), The three-dimensional solar wind around solar maximum, Geophys. Res. Lett., 30(10), 1517, doi:10.1029/ 2003 GL017136.

McComas, D. J., et al. (2006), Ulysses observations of very different heliospheric structure during the declining phase of solar activity cycle 23 , Geophys. Res. Lett., 33, L09102, doi:10.1029/2006GL025915.

McComas, D. J., et al. (2008), Weaker solar wind from the polar coronal holes and the whole Sun, Geophys. Res. Lett., 35, L18103, doi:10.1029/2008GL034896.

McComas, D. J., et al. (2012), The heliosphereŠs interstellar interaction: No bow shock, Science, 336, 1291.

Pryor, W., et al. (2003), Hydrogen atom lifetimes in the three-dimensional heliosphere over the solar cycle, J. Geophys. Res., 108(A10), 8034 doi:10.1029/2003JA009878.

Quemerais, E., and V. Izmodenov (2002), Effects of the heliospheric interface on the interplanetary Lyman alpha glow seen at $1 \mathrm{AU}$ from the Sun, Astron. Astrophys., 396, 269-281.

Quemerais, E., and P. Lamy (2002), Two-dimensional electron density in the solar corona from inversion of white light images - Application to SOHO/LASCO-C2 observations, Astron. Astrophys., 393, 295-304.

Quemerais, E., et al. (2006), Interplanetary hydrogen absolute ionization rates: Retrieving the solar wind mass flux latitude and cycle dependence with SWAN/SOHO maps, J. Geophys. Res., 111, A09114, doi:10.1029/2006JA011711.

Quemerais, E., et al. (2007), Velocity profiles in the solar corona from the multi-instrument observations, Astrophys. J., 667, 1229-1234.

Sokół, J. M., et al. (2012), Heliolatitude and time variations of solar wind structure from in situ measurements and interplanetary scintillation observations, Sol. Phys., 285(1-2), 167-200, doi:10.1007/s11207-0129993-9.

Tobiska, W. K., et al. (2000), The SOLAR2000 empirical solar irradiance model and forecast tool, J. Atmos. Sol. Terr. Phys., 62, 1233-1250.

Tokumaru, M., M. Kojima, and K. Fujiki (2012), Long-term evolution in the global distribution of solar wind speed and density fluctuations during 1997-2009, J. Geophys. Res., 117, A06108, doi:10.1029/2011JA017379. 